Faculdade de Ciências Econômicas UFRGS

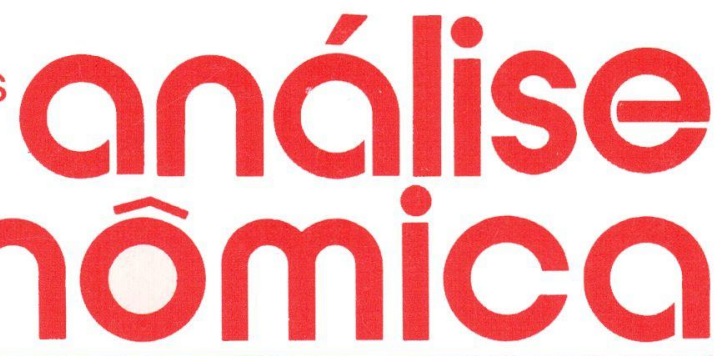

- teoria do balanço de PAGAMENTOS Joáo Luis Tenreiro Barroso

- a IMPORTÂncIa das teorias DE SALÁRIO PARA AS TEORIAS DE INFLAÇÃO Lúcia Maria Góes Moutinho

- os ciclos da economia BRASILEIRA

Vera Beatriz da Silva Oliveira

- SUBSÍDIOS DE PREÇOS AO TRIGO NO BRASIL

Vera Martins da Silva

- A EFICIÊnCIA dA AGRICULtURA GAÚCHA

Juvir Luiz Mattuella

- A economia Regional no BRASIL

Paulo Roberto Haddad

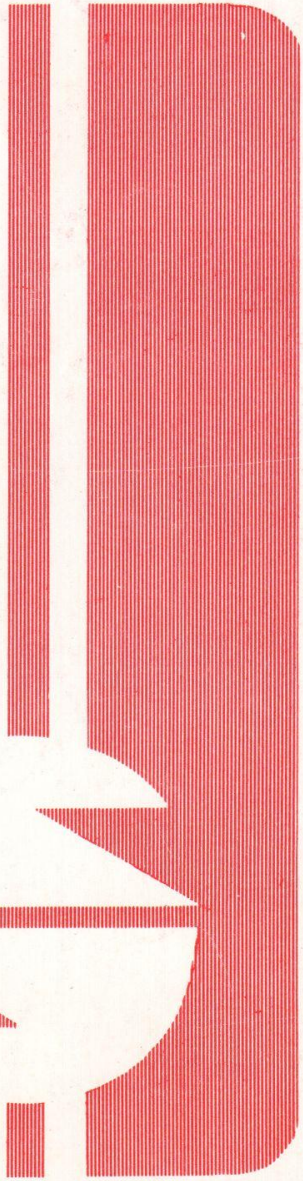


UNIVERSIDADE FEDERAL DO RIO GRANDE DO SUL

Reitor: Prof. Gerhard Jacob

FACULDADE DE CIÊNCIAS ECONÔMICAS

Diretor. Prof. Walter Meucci Nique

CENTRO DE ESTUDOS E PESQUISAS ECONÔMICAS

Diretor: Prof. Reinaldo Ignacio Adams

DEPARTAMENTO DE CIÊNCIAS ECONÔMICAS

Chefe: Profa Oțtlia Beatriz Kroeff Carrion

CURSO DE PÓS-GRADUAÇĀO EM ECONOMIA

Coordenador: Prof. Roberto Camps Moraes

CURSO DE PÓS-GRADUAÇĀO EM ECONOMIA RURAL

Coordenador: Prof. Aray Miguel Feldens

CONSELHO EDITORIAL:

Achyles Barcelos da Costa, Aray Miguel Feldens, Atos Freitas Grawunder, Carlos Augusto Crusius, Ernani Hickmann, João Rogério Sanson, Juvir Luiz Mattuella, Maria Imilda da Costa e Silva, Nali de Jesus de Souza, Nuno Renan Lopes de Figueiredo Pinto, Otília Beatriz Kroeff Carrion, Otto Guilherme Konzen, Paulo Alexandre Spohr, Pedro Cezar Dutra Fonseca, Reinaldo Ignacio Adams, Roberto Camps Moraes, Valter José Stülp, Yeda Rorato Crusius, Edgar Augusto Lanzer (UFSC) e Humberto Vendelino Richeter (UnB)

COMISSĀO EDITORIAL: Atos Freitas Grawunder; Pedro Cezar Dutra Fonseca, Reinaldo Ignacio Adams e Roberto Camps Moraes.

EDITOR: Nali de Jesus de Souza

SECRETARIA: Francisco Carlos Becco dos Santos, Maria Ivone de Mello (normalizaçāo), Vanete Ricacheski (revisão de textos), Wagner Nunes e Zélide Bregaida.

FUNDADOR: Prof. Antônio Carlos Rosa

A Revista ANÁLISE ECONÔMICA publica dois números anuais nos meses de março e novembro. O preço da assinatura, para 1990, é de 7,0 BTNs (o número avulso custa 4 BTNs), a ser pago através de cheque nominal em nome da "Faculdade de Ciências Econômicas/ UFRGS". Aceita-se permuta com revistas congêneres. Aceitam-se, também, livros para elaboração de resenhas ou recensōes.

Toda correspondência, material para publicação, assinaturas e permutas devem ser dirigidos ao seguinte destinatário:

PROF. NALI DE JESUS DE SOUZA

Revista Análise Econômica

Av. João Pessoa, 52 - sala 28

90.040 - PORTO ALEGRE (RS) - BRASIL

Telefone: 25-5844 ramal 34 


\section{RELAÇÃO DAS DISSERTAÇÕES DE MESTRADO DEFENDIDAS E APROVADAS DO CURSO DE PÓS-GRADUAÇÃO EM ECONOMIA RURAL DA UFRGS}

ADAMS, Reinaldo Ignacio. Estrutura e Rentabilidade do Capital nas Empresas Rurais de São Borja, RS. Porto Alegre, IEPE/UFRGS, 1975. 83 f. (Dissertação defendida em 24 de outubro de 1974). Orientador: Humberto Vendelino Richter.

AGOSTINI, Irceu. Alternativas Agrícolas de Inverno em Condições de Risco e de Limitação na Disponibilidade de Miáquinas para a Região do Planalto, Rio Grande do Sul. Porto Alegre, IEPE/ UFRGS. 1981. 92 f. (Tese defendida em 09 de maio de 1980). Orientador: Edgar Augusto Lanzer.

AGUIAR, Deolindo Machado de. Estudo da Viabilidade EconômicoFinanceira de Investimento na Aquisição de Lote Parcelar em Projeto de Irrigação Pública - Caso Bebedouro, Petrolina - PE. Porto Alegre, IEPE/UFRGS, 1989. 264 p. (Dissertação defendida em 17 de julho de 1985). Orientador: Reinaldo Ignacio Adams. Prêmio George Edward Schuh (SOBER). 1986.

ALMEIDA, Alfeu Pinheiro. Análise Econômica de uma Função de Resposta a Calcário e Fósforo na Cultura da Alfafa em Solo de São Jerônimo - RS. (Dissertação defendida em 14 de novembro de 1974). Orientador. Humberto Vendelino Richter.

AMESTOY, Airton Chirivino. Avaliação Econômica das Mudanças de Alguns Coeficientes Técnicos na Ovinocultura de Microrregião Homogênea da Campanha - RS. (Dissertação defendida em 19 de dezembro de 1988). Orientador: Edgar Augusto Lanzer.

ARÊDE, Maria das Graças. O Uso de Mão-de-Obra e Energia em Algumas Culturas do Sul do País, Impactos em sua Utilização Após o Aumento dos Preços de Energia. Porto Alegre, IEPE/ 
UFRGS, 1983. 98 f. (Dissertaçăo defendida em 26 de março de 1982). Orientador: Reinaldo Ignacio Adams.

ARNIZAUT, Paulo. O Abate e o Parque Abatedor de Bovinos no Rio Grande do Sul - 1971/74. Porto Alegre, IEPE/UFRGS, 1977. 144 f. (Dissertação defendida em 26 de maio de 1976). Orientador: Humberto Vendelino Richter.

ASSUMPÇÃO, Antonio Gomes de. A Resposta da Produção Agricola ao Crédito Rural no Estado do Rio Grande do Sul. Periodo 1955/77. Porto Alegre, IEPE/UFRGS, 1983. 122 f. (Dissertação defendida em 26 de março de 1981). Orientador: Edgar Augusto Lanzer.

BAGGIO, Adelar Francisco. Explicação da Renda de Operação Agricola de um Grupo de Agricultores - 1975/76 - Ajuricaba - RS. Porto Alegre, IEPE/UFRGS, 1986. 140 f. (Dissertação defendida em 26 de dezembro de 1978). Orientador. Jorge G. de Oliveira.

BEM, Nilton Pinho de. Tecnologia e Custo de Produção: Análise da Viabilidade da Produção Econômica de Trigo no Rio Grande do Sul. (Dissertação defendida em 26 de abril de 1989). Orientador. Edgar Augusto Lanzer.

BENSUSSAN, Jaques Alberto. Análise Econômico-Financeira dos Efeitos Residuais do Calcário e da Adubação Fosfatada da Cultura do Milho em Três Experimentos do Rio Grande do Sul. (Dissertação defendida em 20 de setembro de 1985). Orientador. Edgar Augusto Lanzer.

BEZERRA, Ildefonso Pinto. Avaliação Econômica sob Condições de Risco de Tecnologias de Produção para a Soja, Milho e Trigo no Rio Grande do Sul. (Dissertaçāo defendida em 14 de julho de 1988). Orientador: Valter José Stülp.

BISCHOFF, Egon Elimar. Efeitos do Uso da Mecanização sobre a Eficiência no Uso de Fatores de Produção em Empresas Agrícolas de Não-Me-Toque (Campo Real) RS. (Dissertaçạo defendida em 24 de julho de 1972). Orientador: Valter J. Stülp.

BORTOLUZI, Clodoaldo Roque Dallajustina. Análise de Fatores Associados à Área e Produtividade do Arroz no Rio Grande do Sul. (Dissertação defendida em 23 de agosto de 1978). Orientador. Humberto Vendelino Richter. 
BÓS, Antonio Miguel Gonçalves. A Produção e o Consumo de Alimentos Básicos no Brasil e a Expansão da Soja. Porto Alegre, IEPE/UFRGS, 1989. 178 p. (Dissertação defendida em 06 de setembro de 1985). Orientador: Aray Miguel Feldens.

BROUWERS, Peter Joseph Antonius. Estudo Descritivo e Análise do Mercado de Ovos de Porto Alegre, RS de 1965/70. Porto Alegre, IEPE/UFRGS, 1972. 189 f. (Dissertação defendida em 14 de maio de 1970). Orientador: Robert Reed.

BUBLITZ, Edmundo Otto. Análise Econômica de Estratégias Alternativas de Banhos Carrapaticidas Através de um Modelo de Simulação. Porto Alegre, IEPE/UFRGS, 1985. 89 t. (Dissertação defendida em 23 de novembro de 1984). Orientador: Edgar Augusto Lanzer.

BUVINICH, Manuel Juan Rojas. Identificação e Análise Econômica de Processos Produtivos Utilizados na Exploração de Novilho Precoce - RS. (Dissertação defendida em 03 de janeiro de 1978). Orientador. Atos Freitas Grawunder.

CAMARGO, Heloisa Helena Tartarotti. Seguro Agrícola contra o Granizo para o Trigo no Rio Grande do Sul. (Dissertaçäo defendida em 23 de agosto de 1976). Orientador. Reinaldo Ignacio Adams.

CARDOSO, Paulo Sérgio. Economia da Seleção de Máquinas e Equipamentos sob Risco - O Caso do Algodão no Norte do Paraná. (Dissertação defendida em 10 de dezembro de 1984). Orientador: Edgar Augusto Lanzer.

CARDOSO, Vera Talita Machado. Elasticidade-Renda da Procura de Alimentos para a Classe de Operários da Indústria de Transformação de Porto Alegre - RS - 1970. (Dissertação defendida em 22 de dezembro de 1972). Orientador. Jorge G. de Oliveira.

CARVALHO JÚNIOR, Luiz Carlos de. Mensuração da Sensibilidade de Alguns Produtos da Lavoura Catarinense aos Principais Instrumentos de Política Agrícola e a sua Rentabilidade Econômica. Porto Alegre, IEPE/UFRGS, 1989.90 p. (Dissertação defendida em 08 de abril de 1986). Orientador: Carlos G.A. Mielitz Netto.

CAVALLAZZI, Mário Roberto. O Desenvolvimento Econômico, o Crédito Rural e o Agente Financeiro. Porto Alegre, IEPE/ 
UFRGS, 1982. 129 f. (Dissertação defendida em 20 de dezembro de 1976). Orientador: Otto Guilherme Konzen.

CEZNE, Wenceslau Miro. Avaliação Econốmica de Métodos Alternativos de Controle da Lagarta da Soja (Anticarsia gemmatalis (Hübner)). Porto Alegre, IEPE/UFRGS, 1989. 96 p. (Dissertaçāo defendida em 05 de julho de 1985). Orientador: Edgar Augusto Lanzer.

COMPARIN, Edelar Luiz. Tipificação das Propriedades Rurais no Estado do Paraná: Subsídios aos Programas de Extensão Rural. Porto Alegre, IEPE/UFRGS, 1988. 201 p. (Dissertaçāo defendida em 18 de dezembro de 1985). Orientador: Otto Guilherme Konzen.

CONCEIÇÃO, Octavio Augusto Camargo. A Expansão da Soja no Rio Grande do Sul de 1950 a 1975. Porto Alegre, IEPE/ UFRGS, 1983. 126 f. (Dissertação defendida em 29 de abril de 1982). Orientador: Otto Guilherme Konzen.

CONTO, Arnaldo José de. Planejamento de Empresas Rurais Típicas em Ibirubá, RS. (Dissertação defendida em 26 de junho de 1972). Orientador: Valter José Stülp.

COSTA, Fernando Paim. Análise Econômica da Produção de Ovos em Condições Comerciais no Rio Grande do Sul. Porto Alegre, IEPE/UFRGS, 1980. (Dissertação defendida em 11 de abril de 1979). Orientador: Edgar Augusto Lanzer.

CUEVAS, Gabriela Ines Riveros. Análise Econômica da Oferta de Pêssegos no Estado do Rio Grande do Sul. Porto Alegre, IEPE/ UFRGS, 1980. 63 f. (Dissertação defendida em 21 de dezembro de 1979). Orientador: Humberto Vendelino Richter.

DAGORD, César José de Leạa. Alocação da Produção de Alimentos e Energia a Partir da Biomassa no Centro Sul do Brasil. (Dissertação defendida em 28 de janeiro de 1987). Orientador: Reinaldo Ignacio Adams.

DIETZE, Sebald Hugo Reckziegel. Viabilidade da Produção Integrada de Cana-de-Açúcar e Álcool Carburante nos Distritos de Obligado, Hohenau, Jesus e Trinidad, Itapuã - Paraguai. (Dissertação defendida em 16 de dezembro de 1987). Orientador: Reinaldo Ignacio Adams. 
DOSSA, Derli. Análise Econĉmica na Aplicação de Nitrogênio, Fósforo e Potássio em um Experimento de Mandioca. Uma Alternativa Metodológica. Porto Alegre, IEPE/UFRGS, 1980. $63 \mathrm{f}$. (Dissertação defendida em 11 de maio de 1979). Orientador: Atos Freitas Grawunder.

ECHEVERRIA, Luis Carlos Robaina. Análise Econômica de Alguns Fatores que Afetam a Capacidade de Amortização de Empréstimos dos Agricultores e Renda da Operação Agricola em Relação ao Uso do Crédito em Carazinho, RS. Porto Alegre, IEPE/ UFRGS, 1973. 143 f. (Dissertaçăo defendida em 31 de agosto de 1972). Orientador: Haralambos Simeonides.

ELY, Aloísio. Análise Econômica da Exploração de Gado de Corte: Alternativas Tecnológicas da Produção Forrageira e Animal, numa Região do Rio Grande do Sul. Porto Alegre, IEPE/UFRGS, 1979. 101 f. (Dissertação defendida em 16 de setembro de 1976). Orientador: Valter José Stülp.

FELDENS, Aray Miguel. Comercialização de Lã no Rio Grande do Sul a Nivel de Cooperativas 1964/1970. (Dissertação defendida em 15 de dezembro de 1972). Orientador: Robert Reed.

FREIRE, Japiassú de Melo. O Efeito do Crédito Rural e de Taxas de Juros Associado à Mudança de Tecnologia, na Renda Líquida nas Pequenas Empresas Rurais - Cruzeiro do Sul - RS. (Dissertação defendida em 16 de abril de 1979). Orientador: Valter José Stülp.

FREIRE, Loiva Maria de Melo. Fatores que Explicam Diferenças de Renda entre Pequenos Produtores Rurais - Cruzeiro do Sul RS. Porto Alegre, IEPE/UFRGS. 1984. 108 f. (Dissertação defendida em 09 de abril de 1979). Orientador: Valter José Stülp.

FREITAS, Claro Luiz de. Mudanças na Posse da Terra e Capitalização da Agricultura no Rio Grande do Sul. (Dissertação defendida em 14 de julho de 1982). Orientador: Otto Guilherme Konzen.

FONSECA, Vera Osório da. Análise Econômica da Aplicação de Doses e Fontes de Nitrogênio na Cultura do Trigo, sob Condições de Risco, em Pelotas, RS. Porto Alegre, IEPE/UFRGS, 1976. 82 f. (Dissertação defendida em 02 de julho de 1976). Orien- 
tador: Edgar Augusto Lanzer. Prêmio "George Edward Schuh" (SOBER). 1976.

GALVÃO, Cláudia Andreoli. A Oferta de Produtos Agrícolas no Rio Grande do Sul: Uma Aplicação do Modelo de Ajustamento Parcial de Marc-Nerlove e Modelo Combinado de Ajustamento Parcial e Expectativas Adaptadas. Porto Alegre, IEPE/UFRGS, 1985. 115 f. (Tese defendida em 05 de agosto de 1980). Orientador: Yeda Rorato Crusius.

GIACOMINI, Nelci Maria Richter. Competitividade e Efeitos da Produção de Cana-de-Açúcar e da Mandioca para Fins Energéticos. (Dissertação defendida em 28 de novembro de 1983). Orientador: Atos Freitas Grawunder.

GIROTTO, Ademir Francisco. Análise da Viaßsilidade Econômica de Diferentes Tipos e Tamanhos de Biodigestores em Uso na Microrregião do Alto Uruguai Catarinense a Nivel de Propriedade Rural. Porto Alegre, IEPE/UFRGS, 1989. 113 p. (Dissertaçāo defendida em 29 de agosto de 1985). Orientador: Valter José Stülp.

GOLLO, Silvana Saionara. A Pequena Produção Agrícola: Alocação de Recursos e Combinação de Atividades nas Pequenas Propriedades do Miunicípio de Erexim - RS. Porto Alegre, IEPE/ UFRGS, 1989. 185 p. (Dissertação defendida em 12 de agosto de 1987). Orientador: Otto Guilherme Konzen.

GOMES, Alalsio Teixeira. Análise dos Custos de Produção de Sulnos em um Grupo de Explorações Localizadas em Microrregiōes Coloniais no Rio Grande dō Sul - no ano de 1973. Porto Alegre, IEPE/UFRGS, 1976. 66 f. (Dissertação defendida em 18 de dezembro de 1975). Orientador: Humberto Vandelino Richter.

GRAWUNDER, Atos Freitas. Planejamento Econômico de uma Fazenda no Rio Grande do Sul. Porto Alegre, IEPE/UFRGS, 1973. 266 f. (Dissertação defendida em 08 de junho de 1971). Orientador: Humberto Vendelino Richter.

GUTIÉRREZ, Júlio Peña. A Mão-de-Obra como Medida do Módulo da Propriedade Rural. Porto Alegre, IEPE/UFRGS, 1966. 112 f. (Dissertação defendida em 20 de abril de 1966). Orientador: Norman Rask.

HOFFMANN, José Hermeto. Fruticultura: Uma Solução para a Baixa 
Renda e o Êxodo Rural da Pequena Propriedade. Porto Alegre, IEPE/UFRGS, 1980. 130 f. (Dissertação defendida em 11 de julho de 1979). Orientador: Atos Freitas Grawunder.

ILHA, Adayr da Silva. Estimação de Relações entre Despesa com Subitens de Alimentação e Despesa Total para Grupos Homogêneos de Famílias da Classe de Operários da Indústria de Transformação de Porto Alegre. Porto Alegre, IEPE/UFRGS, 1983. 126 f. (Dissertação defendida em 20 de julho de 1982). Orientador: José Carlos Grijó.

KITAMURA, Paulo Choji. Economicidade de Investimentos em Conservação de Solos no Sistema Trigo-Soja. Porto Alegre, IEPE/ UFRGS, 1981. 96 f. (Dissertação defendida em 24 de abril de 1981). Orientador: Edgar Augusto Lanzer.

KONZEN, Otto Guilherme. Influência Econômica do Projeto Piloto do Crédito Rural sobre as Empresas Agricolas de lbirubá-RS, Brasil. Porto Alegre, IEPE/UFRGS, 1969. 114 f. (Dissertação defendida em 20 de dezembro de 1968). Orientador: Bernar Lee Erven.

KREUZ, Carlos Leomar. Análise de Tecnologias e Perspectivas da Bovinocultura de Leite na Pequena Propriedade Gaúcha. Porto Alegre, IEPE/UFRGS, 1988. 149 f. (Dissertação defendida em 24 de abril de 1985). Orientador: Atos Freitas Grawunder.

LANDIVAR, Jaime Samuel Solares. Algumas Considerações Econômicas sobre o Transporte da Produção de Cana e de Açúcar do Departamento de Santa Cruz - Bolívia. Porto Alegre, IEPE/ UFRGS, 1985. 178 f. (Dissertação defendida em 18 de abril de 1983). Orientador: Valter José Stülp.

LANZER, Edgar Augusto. Análise Econômica de um Grupo de Experimentos de Fertilização e Calagem do Solo na Cultura do Trigo - Rio Grande do Sul. Porto Alegre, IEPE/UFRGS, 1970. 117 f. (Dissertação defendida em 17 de dezembro de 1969). Orientador: Humberto Vendelino Richter. Prêmio George Edward Schuh (SOBER). 1971.

LEMOS, José de Jesus Sousa. Análise dos Coeficientes Técnicos, dos Custos de Mecanização e das Possibilidades dos Orizicultores Prepararem as Lavouras de Arroz, em Cachoeira do 
Sul e Tapes - RS. (Tese defendida em 16 de julho de 1981). Orientador: Valter José Stülp.

LESSINGER, Egldio. Análise Econômica do Efeito Residual do Fósforo e Calcário num Experimento de Pastagem e Trigo, Através da Função de Produção em Vacaria, RS 1965/70. Porto Alegre, IEPE/UFRGS, 1972. 100 f. (Dissertação defendida em 27 de outubro de 1971). Orientador: Humberto Vendelino Richter.

LIMA, Luiz Mendes de. A Eficiência das Cooperativas Agrícolas Paranaenses na Realização de seus Objetivos Empresariais e Associativos - Modelo e Avaliação. (Dissertação defendida em 24 de setembro de 1976). Orientador: Otto Guilherme Konzen.

LOCATELLI, Antonio. Análise Econômica de Módululos de Organização de Rondônia, O Caso de uma Unidade Típica do Projeto Integrado de Colonização de Ouro Preto. (Dissertaçãao defendida em 19 de novembro de 1986). Orientador: Otto Guilherme Konzen.

LORENCl, Carlos Argeu da Silva. Estrutura das Fazendas e seus Efeitos na Rentabilidade e Capacidade de Amortização de Empréstimos - Alegrete - RS. Porto Alegre, IEPE/UFRGS, 1972. 107 t. (Dissertação defendida em 14 de janeiro de 1971). Orientador: Haralambos Simeonides.

MANSOUR, Abdel Wahad Mohamed Ahmed. Aspectos Econômicos do Mercado Pesqueiro no Estado do Rio Grande do Sul. (Dissertação defendida em 11 de maio de 1976). Orientador: Humberto Vendelino Richter.

MARION FILHO, Pascoal José. A Competitividade e as Repercussões da Produção de Fontes Alternativas de Energia em Pequena Produção nas Comunidades Rurais. (Dissertação defendida em 22 de dezembro de 1987). Orientador: Reinaldo Ignacio Adams.

MASUTTI, Vilson Josø. Estudo das Relações entre Custos, Tecnologia, Nivel de Produção e Escala de Exploração na Pecuária de Leite, Santo Ângelo - RS. Porto Alegre, IEPE/UFRGS, 1989. 93 f. (Dissertação defendida em 12 de setembro de 1984). Orientador. Valter Jos@ Stülp.

MATTUELLA, Juvir Luiz. Análise do Consumo e Renda a Nível de 
Familias Rurais, Campo Real, RS. Porto Alegre, IEPE/UFRG, 1974. 81 f. (Dissertação defendida em 19 de abril de 1974). Orientador: Humberto Vendelino Richter.

MENDES, Judas Tadeu Grassi. Estimativa do Impacto na Renda e na Utilização dos Recursos Devido à Recombinação das Alternativas sob Condições de Restrição no Uso dos Solos do Municipio de Loanda, Estado do Paraná. Porto Alegre, IEPE/ UFRGS, 1976. 83 f. (Dissertação defendida em 22 de março de 1976). Orientador: Humberto Vendelino Richter.

MIELITZ NETTO, Carlos Guilherme Adalberto. Análise das Miudanças de Alguns Coeficientes Técnicos na Criação de Bovinos de Corte no Rio Grande do Sul. Porto Alegre, IEPE/UFRGS. 66 f. (Dissertação defendida em 18 de dezembro de 1978). Orientador: Atos Freitas Grawunder.

MORAES, André Steffens. Dimensionamento Econômico da Frota de Arrasto de Fundo do Rio Grande do Sul. Porto Alegre, IEPE/ UFRGS, 1989. 77 p. (Dissertação defendida em 19 de outubro de 1987). Orientador: Edgar Augusto Lanzer.

MORENO, Pedro Vale. Análise Econômica de Combinações: Máquinas e Implementos e Área das Empresas Rurais do Município de Ibirubá-RS. (Dissertação defendida em 08 de agosto de 1975). Orientador: Juvir Luiz Mattuella.

NADAL, Raul de. Análise Econômica da Recuperação e da Conservação das Condições Fisicas do Solo Santo Ângelo em Lavouras Mecanizadas. (Dissertação defendida em 21 de jultio de 1983). Orientador: Valter José Stülp.

NOGUEIRA JÜNIOR, Sebastião. A Relação Preço-Qualidade e a Procura de Algodão no Reino Unido. Porto Alegre, IEPE/ UFRGS, 1980. 87 f. (Dissertação defendida em 12 de outubro de 1979). Orientador: Peter J.A. Brouwers.

NOSKOSKI, Carlos. Análise Econômica do Uso de Corretivos na Cultura de Trigo. Safra 1969 - Ibirubá - RS. Porto Alegre, IEPE/ UFRGS, 1972. 91 f. (Dissertação defendida em 25 de agosto de 1971). Orientador: Edgar Augusto Lanzer.

NUNES, Evelise Sadonis. Análise Econômica de Produtores Associados a Condomínios Suinícolas: Estudo de Casos - Regional 
de Chapecó-SC-1986. (Dissertação defendida em 01 de fevereiro de 1989). Orientador: Otto Guilherme Konzen.

OLIVEIRA NETTO, José Augusto de. Crédito para Capital de Giro às Cooperativas de Suinocultores no Rio Grande do Sul, Estudo de Casos. (Dissertação defendida em 16 de março de 1970). Orientador. Robert Reed.

ORTIZ, Jaime César Naranjo. Política de Preços e Criação de Emprego na Agricultura Chilena. Porto Alegre, IEPE/UFRGS, 1985. 165 f. (Tese defendida em 26 de junho de 1980). Orientador: Valter José Stülp.

PACHECO, Paulo José. Indicativos da Viabilidade Econômica do Cultivo do Piretro no Rio Grande do Sul - Um Estudo de Casos. (Dissertação defendida em 19 de outubro de 1973). Orientador: Reinaldo I. Adams.

PALMA, Luis. Compatibilidade entre Eficiência Energética e Eficiência Econômica numa Empresa Rural. (Dissertação defendida em 15 de junho de 1983). Orientador: Reinaldo I. Adams.

PAUL, José Maria. Análise Econômica de Funções de Produção em Fertilização e Calagem do Milho na Região do Norte Pioneiro do Paraná. Porto Alegre, IEPE/UFRGS, 1977. 133 f. (Dissertação defendida em 16 de novembro de 1976). Orientador: Peter J. Antonius Browuers.

PEIXOTO, Heverton Rosa. Períodios Ótimos de Venda de Soja Face ao Risco de Mercado. Porto Alegre, IEPE/UFRGS, 1976. $70 \mathrm{f}$. (Dissertação defendida em 31 de maio de 1976). Orientador: Edgar Augusto Lanzer.

PEREIRA, José Maria Dias. A Participação da Alimentação na Inflação Brasileira nos Anos 70 - Uma Contribuição ao Debate. (Dissertação defendida em 09 de dezembro de 1982). Orientador: Aaron Dehter.

PEREIRA, Laércio Barbosa. Estudo da Viabilidade do Incremento nos Niveis de Renda e Emprego nas Pequenas Propriedades Agrícolas do Miunicípio de Torres - RS. Porto Alegre, IEPE/ UFRGS, 1981. 157 f. (Dissertação defendida em 19 de dezembro de 1980). Orientador: Valter José Stülp. 
PÉREZ, José Eduardo Gutiérrez. A Integração Brasil-Argentina: um Estudo da Competitividade na Produção de Grãos. (Dissertação defendida em 09 de dezembro de 1988). Orientador: Valter José Stülp. Prêmio George Edward Schuh (SOBER), 1989.

PIRES, Abelardo Gomes. Alguns Aspectos da Comercialização de Carne Bovina no Rio Grande do Sul. (Dissertação defendida em 06 de fevereiro de 1970). Orientador: Humberto Vendelino Richter.

POLI, João Baptista E. Hofmeister. Descrição e Análise das Rendas em Relação ao Uso de Empréstimos em Pequenas Proprieda-. des Rurais em Lajeado, RS. Porto Alegre, IEPE/UFRGS, 1967. 132 f. (Dissertação defendida em 27 de junho de 1967). Orientador: Roger Johnson.

PONS, José Luiz Marona. Análise Econômica da Alocação de Recursos em um Grupo de Propriedades de Pecuária no Municipio de São Gabriel, RS. Porto Alegre, IEPE/UFRGS, 1975. (Dissertação defendida em 05 de junho de 1974). Orientador: Humberto Vendelino Richter.

PROTAS, José Fernando da Silva. Análise dos Aspectos Econômicos que Envolvem a Cultura de Cebolas a Nível de Propriedade no Município de São José do Norte - RS. Porto Alegre, IEPE/UFRGS, 1980. 60 f. (Dissertação defendida em 11 de dezembro de 1979). Orientador. Atos Freitas Grawunder.

RICHTER, Humberto Vendelino. Produção de Leite em Santa Cruz do Sul - RS. Porto Alegre, IEPE/UFRGS, 1967. 70 f. (Dissertação defendida em 12 de maio de 1964). Orientador: Bueben Buse.

RICHTER, Paulo Ernesto Faillace. Análise Benefício - Custo dos Programas de Crédito Rural. (Dissertação defendida em 21 de outubro de 1983). Orientador: Otto Guilherme Konzen.

RODIGHERI, Honorino Roque. A Adubação Fosfatada e a Competitividade entre as Culturas: Feijão, Milho, Soja e Trigo. Uma Análise Econômica - Cascavel - PR. Porto Alegre, IEPE/ UFRGS, 1980. 90 f. (Dissertação defendida em 09 de maio de 1979). Orientador: Valter José Stülp.

RODRIGUES, Felix Azambuja Contreirás. Análise Econômica Comparativa entre a Bovinocultura de Corte e a de Duplo-Propó- 
sito no RS. Porto Alegre, IEPE/UFRGS, 1989. 143 f. (Dissertação defendida em 30 de maio de 1985). Orientador: Atos Freitas Grawunder.

RODRIGUES, Laerte Pereira. Relações de Custo de Produção de Soja em Sete Municipios Paulistas - Safra 1972/73. (Dissertação defendida em 13 de agosto de 1975). Orientador: Humberto Vendelino Richter.

RODRIGUES, Paulo Coutinho. Análise Econômica de um Sistema de Engorde de Bovino em Confinamento - RS. IEPE/UFRGS, 1975. 96 f. (Dissertação defendida em 28 de novembro de 1973). Orientador: Humberto Vendelino Richter.

ROHR, Ernani João. Investimentos e Custos do Tratamento de Efluentes Poluidores de Curtumes do Vale do Rio dos Sinos RS. Porto Alegre, IEPE/UFRGS, 1983. 170 f. (Dissertação defendida em 12 de agosto de 1982). Orientador: Edgar Augusto Lanzer.

SÁ, José Itamário de. Utilização da Mão-de-Obra e Níveis de Renda em Pequenas Propriedades "Rurais - Santa Rosa, RS. Porto Alegre, IEPE/UFRGS, 1965. 85 f. (Dissertação defendidą em 18 de junho de 1965). Orientador: Norman Rask.

SALLES, Pedro Afonso Almeida de. Análise Econômica de Dois Experimentos de Adubação e Manejo da Pastagem Nativa e sua Implicação na Produtividade da Pecuária. Porto Alegre, IEPE/ UFRGS, 1977. 138 f. (Dissertação defendida em 04 de outubro de 1976). Orientador: Edgar Augusto Lanzer.

SCHUCK, José Hilário. Eficiência no Uso da Terra e das Práticas Agrícolas da Produção de Trigo, Soja e Milho - lbirubá, RS. Porto Alegre, IEPE/UFRGS, 1972. 146 f. (Dissertação defendida em 30 de março de 1971). Orientador: Bernard L. Erven.

SCHWEINBERGER, Geraldo Alzemiro. A Importância das Estruturas de Mercado do Complexo Rural para o Desenvolvimento do Setor Rural. Porto Alegre, IEPE/UFRGS, 1988. 130 f. (Dissertação defendida em 09 de dezembro de 1982). Orientador: Aray Miguel Feldens.

SEABRA, Fernando. A Agricultura e o Desenvolvimento Dualista da Economia do Rio Grande do Sul - 1940-1980. Porto Alegre, 
IEPE/UFRGS, 1989. 96 p. (Dissertação defendida em 26 de novembro de 1985). Orientador: Otto Guilherme Konzen.

SEGALIN, Marlete Maria da Silveira. Uso de Técnica de Transformação de Variáveis Box-Cox em Estimação de Elasticidade-Despesa. (Dissertaçăo defendida em 17 de janeiro de 1989). Orientador. José Carlos Grijo.

SELBACH, Jacob Christiano. A Suinocultura a Nivel de Empresa - Ibirubá, RS. Porto Alegre, IEPE/UFRGS, 1971. 120 f. (Dissertação defendida em 10 de março de 1970). Orientador: Haralambos Simeonides.

SILVEIRA, Custódio Horácio da. Análise Comparativa do Uso da Mecanização em Empresas Rurais - Turvo, Santa Catarina. (Dissertação defendida em 19 de junho de 1975). Orientador: Otto Guilherme Konzen.

SKRABA, Irineu. Função Consumo-Determinação e Análise a Nível de Proprietários Rurais do Municipio de Cruzeiro do Sul - RS. (Dissertação defendida em 03 de setembro de 1979. Orientador: Yeda Rorato Crusius.

SOUZA, Nilson Araújo de. Marcos Conceituais para o Estudo da Repartição da Renda. Porto Alegre, IEPE/UFRGS. (Dissertação defendida em 04 de maio de 1977). Orientador: Aaron Dehter.

SPOHR, Rosani Maria Theobald. Estatuto da Terra, Área Mínima e Programação Linear: uma Aplicação ao Nordeste do Rio Grande do Sul. (Dissertação defendida em 14 de novembro de 1986). Orientador: Edgar Augusto Lanzer.

STEFANELO, Eugênio Libreloto. Análise Econômica e Relação Técnica entre o Rendimento da Soja e o Emprego de Fertilizantes e Calcário em Dez Locais do Rio Grande do Sul. Porto Alegre, IEPE/UFRGS, 1975. 149 f. (Dissertaçăo defendida em 07 de agosto de 1974). Orientador: Humberto Vendelino Richter.

STÜLP, Valter José. Planejamento de uma Empresa Rural Típica em Estrela, RS. Pọto Alegre, IEPE/UFRGS, 1971.218 f. (Dissertação defendida em 12 de outubro de 1970). Orientador: Haralambos Simeonides. 
TALAMINI, Dirceu João Duarte. Análise Econômica dos Sistemas de Produção de Suínos - Santa Catarina. Porto Alegre, IEPE/ UFRGS. (Dissertação defendida em 29 de setembro de 1977). Orientador: Valter José Stülp.

TOMASINI, Roque Gilberto Annes. Influência dos Prazos e Condições dos Contratos na Produtividade Agrícola e Econômica dos Arrendatários e Parceiros, Carazinho e Não-Me-Toque (Campo Real), RS em 1970. (Dissertação defendida em 25 de setembro de 1971). Orientador: Humberto Vendelino Richter.

TONERA, Hélcio. A Influência do Crédito e da Assistência Técnica sobre as Propriedades Rurais de Timbó e Cedros, Sta. Catarina. (Dissertação defendida em 31 de outubro de 1969). Orientador: Otto Guilherme Konzen.

TORESAN, Luiz. Tecnologia e Rentabilidade na Agricultura: Um Estudo de Caso. Porto Alegre, IEPE/UFRGS. 268 p. (Dissertação defendida em 13 de novembro de 1987). Orientador: Edgar Augusto Lanzer. Prêmio George Edward Schuh (SOBER). 1988.

UHLIG, Lourival. Análise Econômica de Sistemas Alimentares para a Produção Suína, a Nivel de Propriedade Rural. (Dissertação defendida em 05 de fevereiro de 1986). Orientador: Valter José Stülp.

VARASCHIN, Vitốrio Manoel. Microdestilaria - Uma Estratégia para Desenvolvimento Rural. (Dissertaçäo defendida em 21 de dezembro de 1982). Orientador. Reinaldo l. Adams.

VILANI, Daniel. Avaliação de Possibilidade de Auto-Suficiência Energética a Nível de Comunidade. Porto Alegre, IEPE/UFRGS, 1984. 120 f. (Dissertação defendida em 09 de dezembro de 1983). Orientador: Reinaldo Ignacio Adams. Prêmio George Edward Schuh (SOBER). 1984.

VILCA HUAMÁN, Luciano Javier Montoya. Experimento na Cultura do Milho: Análise Econômica e Custos de Decisões sob Incerteza. Porto Alegre, IEPE/UFRGS, 1979. 99 f. (Dissertação defendida em 06 de junho de 1977). Orientador: Peter J.A. Brouwers.

WESTPHAL, Selmo. Tendências da Produção e Produtividade Agrícola e Modificações no Uso dos Fatores Terra, Mão-de-Obra e Mecanização, Estado do Paraná - 1960/70. Porto Alegre, IEPE/ 
UFRGS, 1976. 116 f. (Dissertação defendida em 28 de novembro de 1975). Orientador: Otto Guilherme Konzen.

WEYDMANN Celso Leonardo. Análise Econômica da Citricultura na Pequena Propriedade Rural. Porto Alegre, IEPE/UFRGS, 1989. 172 f. (Dissertação defendida em 04 de janeiro de 1984). Orientador: Atos Freitas Grawunder.

WILGES, IImo José. O Impacto das Medidas Governamentais sobre Pequenas Propriedades Agricolas - Lajeado/RS - 1965/69. (Dissertação defendida em 29 de outubro de 1976). Orientador: Otto Guilherme Konzen.

ZAGATTO, Luiz Carlos Ayres Guidetti. Mudanças Ocorridas no Processo Produtivo de Pequenas Propriedades Agrícolas - Cruzeiro do Sul, RS - 1972/76. (Dissertação defendida em 11 de setembro de 1979). Orientador: Atos Freitas Grawunder.

ZUNG, Che Yee. Função dos Sistemas Administrativos e sua Eficácia - Cooperativas Agricolas do Paraná. (Dissertação defendida em 28 de junho de 1979). Orientador: Otto Guilherme Konzen.

NOTA: As dissertaçōes de Economia Rural, acima relacionadas, assim como as dissertaçōes de Economia, arroladas no número 10, de novembro de 1988, encontram-se à đisposição, para consulta, na Biblioteca da Faculdade de Ciências Econômicas da Universidade Federal do Rio Grande do Sul. 